EPJ Web of Conferences 21, 09004 (2012)

DOI: $10.1051 /$ epjconf/20122109004

(C) Owned by the authors, published by EDP Sciences, 2012

\title{
Iwamoto-Harada model of pre-equiluibrium cluster emission: Should we care about angular momentum?
}

\author{
Emil Běták ${ }^{1,2, a}$ \\ 1 Institute of Physics, Slovak Acad. Sci., 84511 Bratislava, Slovakia \\ 2 Inst. of Physics, Fac. of Philosophy and Sci., Silesian Univ. 74601 Opava, Czech Rep.
}

\begin{abstract}
Whereas the emission of nucleons in low-energy nuclear reactions (say, below the pion threshold) can be nicely described using statistical models (compound nucleus plus pre-equilibrium), that of the complex particles, i.e. light clusters up to $\alpha$ 's, is far from satisfactory state. The Iwamoto-Harada model of pre-equilibrium cluster emission was formulated within spin-independent version of the exciton model. The inclusion of angular momentum into the pre-equilibrium reactions proved to be important and essential for the $\gamma$ emission. The angular-momentum couplings have not yet been applied to the light cluster emission; however, the connection with deformation suggested by Blann has been shown to have visible effects. Our study is aimed to consider, whether and how the angular-momentum couplings influence the light cluster emission within the IwamotoHarada model.
\end{abstract}

\section{Introduction}

We use the pre-equilibrium coalescence exciton model as a basis for our approach to the statistical description of light cluster (or complex particles, i.e. deuterons to alphas) emission at energies of several tens of $\mathrm{MeV}$ (see [1,2]). The coalescence models [3] are of more general nature that the preformed ones [4] and they are currently applied to all types of light complex particles. Apart from these two groups of models with straightforward physical backgrounds, phenomenological descriptions are also popular $[5,6]$. In fact, their predictive power is higher than those of the former group, though they are handicapped by more parameters.

The exciton model assumes the reaction to proceed via a sequence of states characterized by their exciton number (see [1]). The energy spectrum of the emitted particles in the spin-independent formulation of the model is

$$
\frac{\mathrm{d} \sigma}{\mathrm{d} \varepsilon_{x}}=\sigma_{\mathrm{R}} \sum_{n} \tau_{n} \lambda_{x}^{c}\left(n, E, \varepsilon_{x}\right),
$$

where $\lambda_{x}^{c}\left(n, E, \varepsilon_{x}\right)$ is the particle emission rate from an $n$-exciton state $(n=p+h)$ of excitation energy $E$ to continuum, the energy of the ejectile of type $x$ is $\varepsilon_{x}$, and $\tau_{n}$ and $\sigma_{\mathrm{R}}$ are the time spent in an $n$-exciton state and the cross section of creation of the composite system, respectively ${ }^{1}$.

The nucleon ( $x$ stands for proton $\pi$ or neutron $v$ ) emission rate (see, e.g. [1]) is

$$
\lambda_{x}^{c}\left(n, E, \varepsilon_{x}\right)=\frac{2 s_{x}+1}{\pi^{2} \hbar^{3}} \mu_{x} \varepsilon_{x} \sigma_{\mathrm{INV}}^{*}\left(\varepsilon_{x}\right) \frac{\omega(p-1, h, U)}{\omega(p, h, E)} R_{x}(p),
$$

\footnotetext{
a e-mail: betak@savba.sk

1 Some authors prefer using closed expressions. In such case, the summation in (1) stops at the equilibrium exciton number $\bar{n}$, and the compound nucleus contribution (reduced by the pre-equilibrium emission) is added to the truncated sum.

This is an Open Access article distributed under the terms of the Creative Commons Attribution-Noncommercial License 3.0, which permits unrestricted use, distribution, and reproduction in any noncommercial medium, provided the original work is properly cited.
} 


\section{EPJ Web of Conferences}

where $\mu_{x}$ and $s_{x}$ are the ejectile reduced mass and spin, respectively, $\sigma_{\mathrm{INV}}$ is the inverse cross section, which is, in fact, replaced by the cross section of the capture of a projectile $x$ by the nucleus in its ground state, and $U$ is the energy of residual nucleus which is produced in an $(n-1)$-exciton state. The factor $R_{x}(p)$ takes into account the proton-neutron composition. In fact, it stands as an effective quantity which arises from replacing the two-component description (i.e. that distinguishing between the neutrons and the protons) by an one-component one. There are several ways of introducing the charge factor. We follow here the charge factor $R_{x}(p)$ of [3]. Explicitly, $R_{\pi}(p)+R_{v}(p)=1$ holds for nucleons at each stage of the reaction. Anyway, none of different suggested forms of charge factors has proper behaviour both at the very early stage of the reaction process and at equilibrium, and similarly these factors cannot withstand the detailed balance principle (see also [7, 1]).

\subsection{General parameters of the model}

The most pronounced parameters of the exciton model are the initial exciton number $n_{0}$ and the average squared transition matrix element $|M|^{2}$. The intensity of the intranuclear transitions may be also expressed using the optical potential, if we allow for scaling by some factor.

Though the matrix element $|M|^{2}$ can be, in principle, derived from nuclear matter studies, the values needed to reproduce observed cross sections differ significantly from those obtained from the basic knowledge. Therefore, some of the parametrized forms are used, e.g. $|M|^{2}=K^{\prime} \cdot A^{-3} e^{-1}$, where $e=E / n$ (c.f. [8]). The value of $K^{\prime}=100-110 \mathrm{MeV}^{3}$ gives reliable overall results at energies below $40 \mathrm{MeV}$, where reasonable amount of data is available [9].

The initial exciton number $n_{0}$ can be obtained from the analyses of the slopes of particle energy spectra at their high-energy edge (see [10]). Simple arguments would lead to $n_{0}=A_{\text {proj }}$ for light-projectile induced reactions (at least on even-even targets) $[3,11,12]$, which implies $n_{0}=1$ for nucleon- and $n_{0}=4$ for $\alpha$-induced reactions, respectively ${ }^{2}$. Obviously, target structure and the Coulomb interaction between the target and the projectile may cause partial departures from this simple rule.

\section{Cluster emission}

\subsection{Cluster coalescence}

In the most simple case, the cluster emission rate can be written formally in exactly the same way as it was for nucleons, just with replacing the exciton number of the residual nucleus $(p-1, h)$ by $\left(p-p_{x}, h\right)[3]^{3}$, where we assume that the cluster is formed by $p_{x}$ of the total of $p$ excited particles (see also Fig. 1). The original form of the coalescence model [3] has been soon improved by Ribanský and Obložinský [14] who improved the approach [3] by replacing the artificial $p_{x}$ ! factor by $\gamma_{x} \times$ $\omega\left(p_{x}, 0, \varepsilon_{x}+B_{x}\right) / g_{x}$, which has straightforward physical interpretation: its second part is the number of configurations of the $p_{x}$ excitons, and $\gamma_{x}$ is the formation probability, which contains all the dynamics of the process. In practice, however, the single-cluster density $g_{x}$ is often replaced by the single-particle one ${ }^{4}$. Thus, the emission rate $[14]$ reads

$$
\begin{array}{r}
\lambda_{x}^{c}\left(n, E, \varepsilon_{x}\right)=\frac{2 s_{x}+1}{\pi^{2} \hbar^{3}} \mu_{x} \varepsilon_{x} \sigma_{\mathrm{INV}}^{*}\left(\varepsilon_{x}\right) \frac{\omega\left(p-p_{x}, h, U\right)}{\omega(p, h, E)} \\
\times \gamma_{x} \frac{\omega\left(p_{x}, 0, \varepsilon_{x}+B_{c}\right)}{g_{x}} R_{x}(p) .
\end{array}
$$

\footnotetext{
2 The slope analyses prefer $n_{0}=3$ in the former case, but as no emission (up to a tiny correction due to the $\gamma$ emission) can proceed from the $n=1$ state, its whole strength is transferred to the 3 -exciton one, which is therefore "seen" as the first emitting stage in the slope analysis.

${ }^{3}$ In fact, this idea appeared already in the paper of Blann and Lanzafame [13]. However, their predicted complex particle spectra were deeply below the experimental data.

${ }^{4}$ See also the discussions of the role of the single-cluster density $[15,16]$.
} 


\section{$\mathrm{CNR} * 11$}
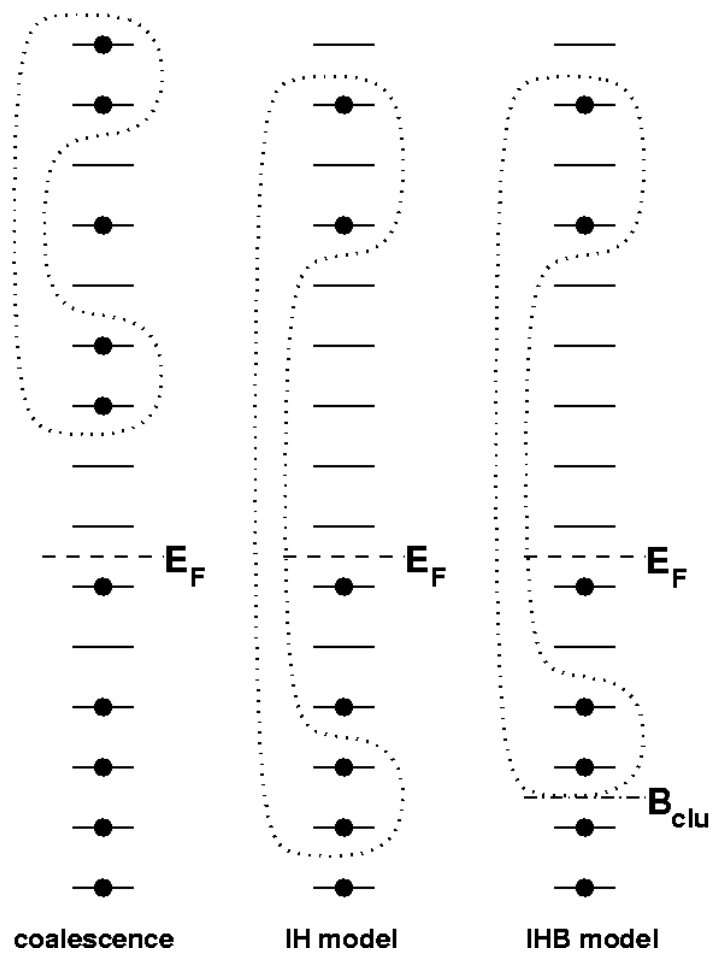

Fig. 1. Schemes of coalescence and related mechanisms of the cluster pre-equilibrium emission (all parts drawn for the case of the $\alpha$ emission here). The very left one is the pure coalescence process, when the cluster $(\alpha$ particle) is composed of the excited particles. The number of the excited particles must be higher or equal to the mass number of the ejectile. The second column is the so-called Iwamoto-Harada mechanism, i.e. the statistical equivalent of pickup: The cluster is formed from both the excited particle excitons and the ones picked-up from the Fermi sea. The third column depicts the Bisplinghoff improvement of the Iwamoto-Harada model: the layer of the unexcited particles available for the pickup process is limited by the binding energy of the nucleons in the cluster. Finally, the most right picture is a knockout: all nucleons are knocked out from the unexcited states.

This approach led to both reasonable absolute values and for some clusters even rather good spectra shapes. A commonly used approach is to consider $\gamma_{x}$ to be a parameter obtainable from the fit to the data. Typical values are of the order of $10^{-3}$ for $\alpha$ 's and of $10^{-2}$ for deuterons.

Simple theoretical estimates of the formation probability of the coalescence model yield e.g. $\gamma_{x} \approx$ $p_{x}{ }^{3}\left(p_{x} / A\right)^{p_{x}-1}[17]$ or $\gamma_{x}=\left[\frac{4}{3}\left(P_{0} / m c\right)^{3}\right]^{p_{x}-1}$ [18], where $P_{0}$ is the radius of the sphere in the momentum space containing the nucleons which are picked up and it is found to be close to the Fermi momentum, or to $\gamma_{x} \approx 1 / A^{m}$ above mass 27 , with $m \approx 1$ for deuterons and $m \approx 4 / 3$ for $\alpha$ 's, tritons and ${ }^{3} \mathrm{He}$ [19]. However, the presence of formation probabilities and/or other additional functions is not strictly justifiable by detailed balance, and it is therefore rejected by some groups (e.g. [5, 20,21]), even though it means worsening of the agreement between theory and data.

\subsection{Pre-Formed alphas}

The approach of the Milano group of pre-formed $\alpha$ emission [4] uses the fact that the $\alpha$ particle is a very strongly coupled object, whose existence (as a cluster) has been frequently demonstrated in nuclear studies. Thus, it need not be formed in the course of a reaction, and may enter the process as a pre-formed entity, which can be considered as a single exciton, though of properties (mass, spin, etc.) specific for the $\alpha$ particle. This approach is not a general one for different clusters, but it has been formulated solely for the case of the $\alpha$ emission. Instead of formation probability we have now 


\section{EPJ Web of Conferences}

the preformation factor $\gamma_{\alpha}$, which is the probability that $\alpha$ is present in the target and that it becomes involved in the reaction by interaction of the projectile with the target. The probability (pre-formation factor) $\gamma_{\alpha}$ enters the emission rates, which are (apart from this factor) formally identical to those for the nucleon emission.

\section{The Iwamoto-Harada (pickup) model}

The coalescence model has been made more sophisticated to allow the cluster to be formed not only from excitons, but also from unexcited nucleons below the Fermi level (see the second column in Fig. 1). This approach became known as the Iwamoto-Harada model [22] ${ }^{5}$, even though it has been suggested and applied five years earlier [15]. The main difference of both formulations [15,22] is just a technical aspect: whereas Iwamoto and Harada [22] calculated the clusterization in the momentum space, the formaer paper [15] used the energy space, what is fully consistent with underlying ideas of the exciton model. Mathematically, the switch from pure coalescence to the Iwamoto-Harada model (in formulation of [15]) means replacing of the density product $\omega\left(p-p_{x}, h, U\right) \times \omega\left(p_{x}, 0, \varepsilon_{x}+B_{x}\right)$ by

$$
\sum_{p^{*}=1}^{p_{x}} \int_{\varepsilon_{x}+B_{x}}^{E} \omega\left(p-p^{*}, h, E-\varepsilon_{1}\right) \omega\left(p^{*}, 0, \varepsilon_{1}\right) \omega\left(0, p_{x}-p^{*}, \varepsilon_{2}\right) \mathrm{d} \varepsilon_{1},
$$

where $p^{*}$ is the number of (particle) excitons contributing to form the cluster (their energy is $\varepsilon_{1}$ ), and the remaining $\left(p_{x}-p^{*}\right)$ nucleons $i$ are picked up from the Fermi sea. Now, the cluster density is $g_{x}=\gamma_{x} \cdot g\left[g\left(\varepsilon_{x}+B_{x}+p_{x} E_{F}\right)\right]^{p_{x}-1} /\left[p_{x} !\left(p_{x}-1\right) !\right]$, making this approach parameterless for cluster emission [15].

In the case of the nucleon emission, the process leads from a $(p, h)$-state in the composite system to $(p-1, h)$-state in the residual one, and the coalescence model in its original formulation leads to $\left(p-p_{x}, h\right)$ in the process of the cluster emission. The Iwamoto-Harada approach reaches $\left(p-p^{*}, h+p^{*}\right)$ as its final state, where $p^{*}$ takes all values from 1 to $p_{x}$. This also implies that the cluster emission can proceed already from more simple initial states than those necessary for the emission of cluster of $p_{x}$ nucleons in the pure (original) coalescence model ${ }^{6}$

Bisplinghoff suggested (we use IHB to denote the Iwamoto-Harada model with Bisplinghoff's generalization) that not all nucleons be available for the cluster formation within the model, but only those close to the Fermi energy, and the energy width of the "band of availability" is determined by the binding energy of nucleons inside the cluster [23]. It is natural to generalize the idea to arbitrary combinations of excited and unexcited nucleons, and to all types of clusters. As the binding energy of nucleons in the deuteron is small, the pick-up possibility is hardly likely to be observed in practice. Thus, strongly bound entities, like $\alpha$ 's, have large energy space available for their creation (which makes the approach close to the original [22,15] ideas, and loosely coupled objects (e.g. deuterons) practically get close to the standard coalescence model [24,25].

\section{Improvements of the model}

In order to improve the versatility of the Iwamoto-Harada model, several additions have been introduced still into its spin-independent formulation. Essentially, they are: $i$ ) generalization of the Bisplinghoff idea to other types of clusters, not only to $\alpha$ 's; ii) consider possible blurring of energy levels

\footnotetext{
5 We list only the initial paper by Iwamoto and Harada here, and not the relevant subsequent ones.

6 The form of the emission spectra stems from different emission stages (different exciton numbers). However, at the high-energy edge of the spectrum, the contributions from complex later stages become negligible and the emission is determined by the initial (emitting) exciton configuration, so that $\mathrm{d} \sigma_{x} / \mathrm{d} \varepsilon_{x} \propto f\left(\varepsilon_{x}\right) U^{n_{0}-\Delta_{n}}$, where $n_{0}$, $\Delta_{n}$ and $f\left(\varepsilon_{x}\right)$ depend on the model assumptions of clusterization. The original form has been written for nucleons by Blann [10], later generalized to the cluster emission [26]. Therefore, the calculations are extremely sensitive to the mechanisms assumed to play role at the very initial steps of the process.
} 


\section{$\mathrm{CNR} * 11$}

as a result of thermal motion of nucleons within the nucleus; iii) ensuring that the pre-equilibrium cluster formulae are capable to reach proper equilibrium (compound nucleus) limit - this implies a transition from Iwamoto-Harada process to pure coalescence one in the course of the reaction; $i v$ ) introducing energy uncertainty due to extremely short rapid stages at the beginning of a reaction; $v$ ) considering possibility of knock-out for the most coupled ejectile, i.e. for the $\alpha$ particle at least (fortunately, it can be expressed using the language of the exciton model!).

These modifications were introduced to the pre-equilibrium decay at the Santa Fe Nuclear Data conference [27], and the agreement to the data improved significantly.

\section{Angular momentum}

\subsection{Angular distributions}

Current pre-equilibrium models often ignore the influence of angular momentum. This is easily shown to be rather small for the nucleon emission, but is larger for clusters. The effect arises from: $i$ ) cluster emission is usually enhanced at higher angular momenta, which means increased role of the nuclear surface and consequently effective lowering of the Coulomb barrier, especially in the case of deformed nuclei; ii) many of quantities entering the pre-equilibrium reactions are both spin- and energydependent, and their simple contraction to one variable necessarily affects the results. The consistent incorporation of the angular momentum is more complicated. Some steps have been undertaken in [28], but the full formulation of the spin-dependent intranuclear transition and emission rates has been enabled by Obložinský [29] and incorporated into the code PEGAS [30]. However, clusters are not included therein yet.

Initially, pre-equilibrium models were used to describe the energy spectra of the emitted particles. The angular distributions have been added using the idea of a fast (leading) particle [31]: in nucleoninduced reactions, the original exciton (the incident nucleon) is assumed to be the most energetic one and it keeps the notion of its direction, which is slowly smeared out in the course of the reaction. The nucleon-nucleon differential cross section is system it is proportional to $\cos \theta \times \Theta\left(\frac{\pi}{2}-\theta\right)$, where $\theta$ is the angle measured with respect to the original direction. With each interaction, the angular distribution gets consequently flatter.

A general way for the cluster angular distributions has been suggested by considering the linear momentum shared by all the excitons together [32,33]. The approach was much improved in [34], together with a method to calculate the densities with specified linear momentum.

A popular way to express the angular distributions within the exciton (or hybrid) model(s) uses the systematics [35] including purely phenomenological approaches with the contribution to the cross section both due to the pickup/stripping and knockout and inelastic processes approximately considered (see the original papers $[5,35]$ ). These approaches are applied to wider range of projectiles and ejectiles and the approach is currently used in many computer codes today.

\subsection{Deformation}

Rather often the deformation effects are not considered in the pre-equilibrium decay. It was shown by Blann [36] that taking deformation into account strengthens especially the cluster emission. However, this approach has not called much attention.

\subsection{Spin variables in the exciton model}

Incorporation of spin variables into the pre-equilibrium exciton model [29,34] has been developed for the equilibration process, nucleon and $\gamma$ emissions. Obviously, if we calculate the time spent in the $n$-exciton state by solving the set of master equations [30], this set becomes much larger (from tens of thousands coupled equations up to millions of them). We do not repeat the spin-coupling formulae here, but refer to the original paper [29] and its realization in the computer code DEGAS [30]. 

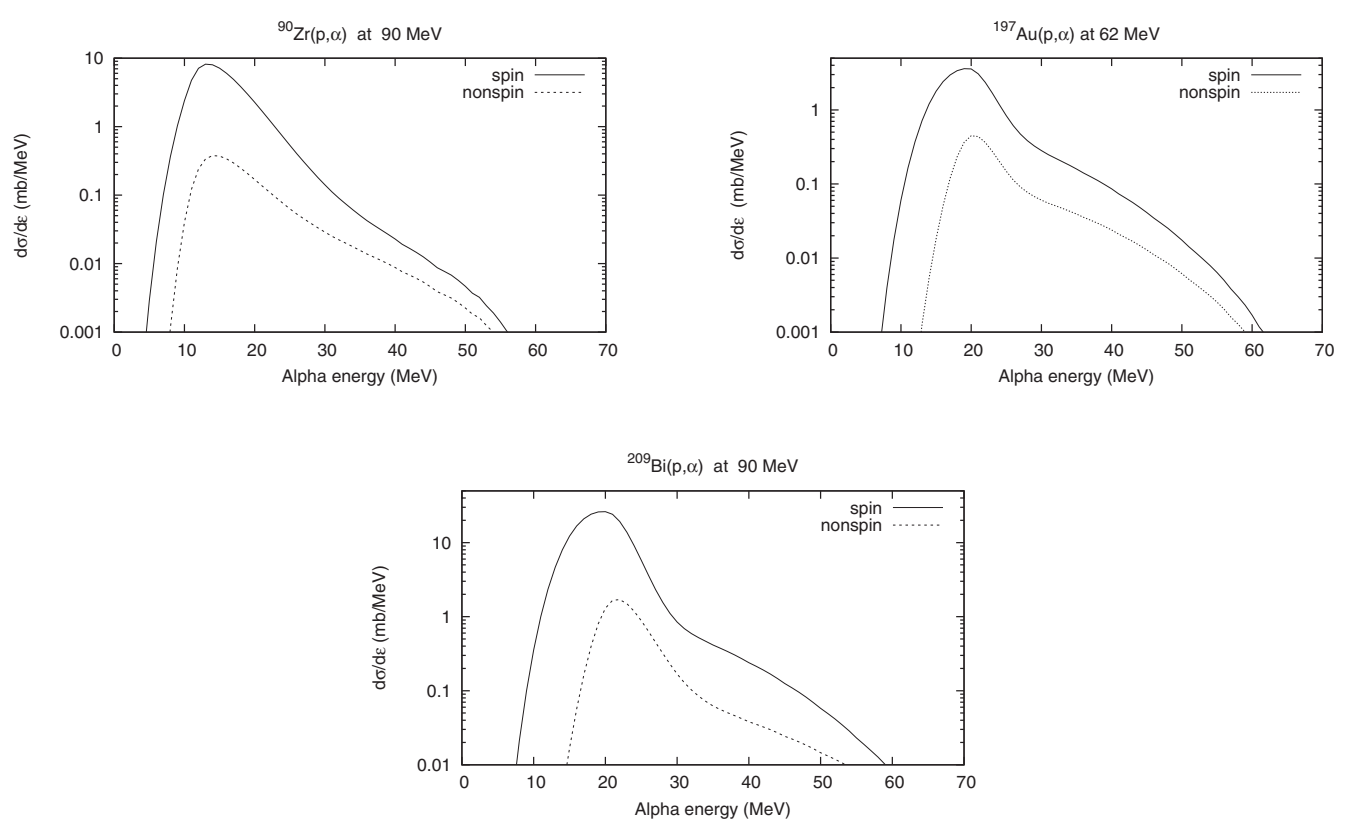

Fig. 2. Angle-integrated $\alpha$ energy spectra from three proton-induced reactions on ${ }^{90} \mathrm{Zr},{ }^{197} \mathrm{Au}$ and ${ }^{209} \mathrm{Bi}$ at 62 $\mathrm{MeV}(\mathrm{Au})$ or $90 \mathrm{MeV}$ (two other reactions). The full curves represent the calculations with angular momentum, whereas the dotted ones are without.

The question arises, how to apply these couplings to the cluster emission? For a first view, similar (but more complicated) set of formulae should be derived here and consequently used in a computer code. However, one can - at least as a good approximation - use the fact the the formation probability $\gamma_{x}$ contains the dynamics of the process, i.e. also the spin couplings. We should emphasize that such happy coincidence of canceling the cluster couplings is possible for the Iwamoto-Harada model formulated in the exciton-energy space, and it cannot be applied to the cases, where the final expression for the cluster emission contains the formation probability or some similar quantity. This essentially simplifies the task here and makes it feasible to be applied to calculations of nuclear reactions. We have adopted this way and we present the basic results below.

\section{Calculations}

For the calculations, we have chosen three $(\mathrm{p}, \alpha)$ reactions, which have been already calculated in the spin-independent case $[27,37]$, namely on ${ }^{90} \mathrm{Zr},{ }^{197} \mathrm{Au}$ and ${ }^{209} \mathrm{Bi}$. In order to show the angular momentum influence unscreened by other effects, we have abstracted from the improvements to the Iwamoto-Harada model now and even we use default level density parameters, i.e. $g=A / 13$ and no pairing here. Thus, the calculated energy spectra are not aimed to be compared to the data, as the parameters of the model are not "tuned" for that, but simply to illustrate the influence of angular momentum newly introduced for the pre-equilibrium cluster emission. Fig. 2 presents the energy spectra for all three reactions, calculated both with and without angular momentum. The essential pre-equilibrium parameters, as the level density ones or the transition matrix element, are kept identical in both sets of calculations. Only the first emitted particle is calculated so that they do not include any consequent emissions.

In all cases, the introduction of angular momentum increases the cluster spectra significantly and somewhat lowers the emission of nucleons (as the reaction cross section $\sigma_{\mathrm{R}}$ is the same both with and without angular momentum, and it has to be split among neutron, proton, cluster and $\gamma$ emissions). 


\section{$\mathrm{CNR} * 11$}

Thus we arrive to result in a similar direction as was obtained by Blann by considering deformed nuclei.

\section{Conclusions}

We have applied the angular momentum couplings to express the light cluster emission within the pre-equilibrium exciton model formalism. This inclusion increases the cluster emission at the initial stages of a reaction (and due to particle competition) it lowers that of nucleons.

The calculations are only at their initial stage, and usage of carefully selected level density parameters and of consequent particle emission within this formalism should be only done.

\section{Acknowledgments}

The author is grateful to J. Dobeš and P. Obložinský for discussions. The work has been supported in part by the VEGA Grant No. 2/0029/10.

\section{References}

1. E. Gadioli, P.E. Hodgson, Pre-Equilibrium Nuclear Reactions, (Clarendon Press, Oxford 1992) and the references quoted therein.

2. P.E. Hodgson, E. Běták, Phys. Rep. 374, 1 (2003)

3. C.K. Cline, Nucl. Phys. A 193, 417 (1972)

4. L. Milazzo-Colli, G.M. Braga-Marcazzan, Phys. Lett. B 36, 447 (1971); ibid. Phys. Lett. B 38, 155 (1972); G.M. Braga-Marcazzan, L. Milazzo-Colli, C. Signorini, Lett. N. Cim. 6 (1973), 357 (1973)

5. C. Kalbach, Z. Phys. A 283, 401 (1977)

6. C. Kalbach, Phys. Rev. C 71, 034606 (2005)

7. E. Běták, P.E. Hodgson, Rep. Prog. Phys. 61, 483 (1998)

8. C. Kalbach, Z. Phys. A 287, 319 (1978)

9. F. Cvelbar, E. Běták, Z. Phys. A 332, 163 (1989); F. Cvelbar, E. Běták, J. Merhar, J. Phys. G 17, $113(1991)$

10. M. Blann, Annu. Rev. Nucl. Sci. 25, 123 (1975)

11. D. Agassi, H.A. Weidenmüller, G. Mantzouranis, Phys. Rep. C 22, 145 (1975)

12. E. Gadioli, E. Gadioli Erba, L. Sajo-Bohus, G. Tagliaferri, Riv. N. Cim. 6, 1 (1976)

13. M. Blann, F.M. Lanzafame, Nucl. Phys. A 142, 559 (1970)

14. I. Ribanský, P. Obložinský, Phys. Lett. B 45, 318 (1973)

15. J. Dobeš, E. Běták, Proc. International Conf. Reaction Models '77, Balatonfüred 1977 (ed. L.P. Csernai; Budapest), p. 195 (1977)

16. P. Obložinský, I. Ribanský, Phys. Lett. B 74, 6 (1978)

17. E. Běták, P. Obložinský, Proc. 5th International Symposium Interactions of Fast Neutrons with Nuclei, Gaussig 1975, Report ZfK-324 (ZfK, Rossendorf), p. 77 (1976)

18. H. Machner, Phys. Lett. B 86, 129 (1979)

19. J.R. Wu, C.C. Chang, Phys. Rev. C 17, 1540 (1978)

20. C. Kalbach, Phys. Rev. C 19, 1547 (1979)

21. C. Kalbach, Phys. Rev. C 37, 2350 (1988)

22. A. Iwamoto, K. Harada, Phys. Rev. C 26, 1821 (1982)

23. J. Bisplinghoff, Phys. Rev. C 50, 1611 (1994)

24. E. Běták, Proc. 9th International Conf. on Nuclear Reaction Mechanisms, Varenna 2000 (ed. E.

Gadioli; Ric. Sci. Educ. Perman., Suppl. No. 115, Univ. Milano), p. 591 (2000)

25. E. Běták, Int. J. Mod. Phys. E 13, 63 (2004) 
26. E. Běták Collective Motion and Giant Resonances. Proc. 15th Mikolajki Summer School Nucl. Phys., Mikolajki 1983. Seminars. (eds. Z. Wilhelmi and M. Kicińska-Habior; Warsaw University) p. 132 (1984)

27. E. Běták, AIP Conf. Proceedings, vol. 769. Amer. Inst. Phys., Melville and New York, 2005, p. 1168

28. Shi Xiangjun, H. Gruppelaar, J.M. Akkermans, Nucl. Phys. A 466, 333 (1987)

29. P. Obložinský, Phys. Rev. C35, 407 (1987); P. Obložinský, M.B. Chadwick, ibid. C42, 1652 (1990)

30. E. Běták, P. Obložinský, INDC(SLK)-001 (IAEA Vienna 1993) and its later developments (unpublished)

31. G. Mantzouranis, D. Agassi, H.A. Weidenmüller, Phys. Lett. B 57, 220 (1975); G. Mantzouranis, H.A. Weidenmüller, D. Agassi, Z. Phys. A 276, 145 (1976)

32. P. Mädler, R. Reif, Nucl. Phys. A 337, 445 (1980), 445; P. Mädler, R. Reif, V. D. Toneev, Nucl. Phys. A 373, 27 (1982)

33. K.K. Gudima, S.G. Mashnik, V.D. Toneev, Nucl. Phys. A 401, 329 (1983)

34. M. B. Chadwick, P. Obložinský, Phys. Rev. C 44, R1740 (1991) M. B. Chadwick, P. Obložinský, Phys. Rev. C 46, 2028 (1992)

35. C. Kalbach, F. M. Mann, Phys. Rev. C 23, 112 (1981); ibid. 23, 124 (1981)

36. M. Blann, Phys. Rev. C 21, 1770 (1980); M. Blann, T.T. Komoto, Phys. Rev. C 24, 426 (1981)

37. E. Běták, Proc. XXIV Internat. Workshop on Nuclear Theory, (Rila Mountains, Bulgaria, June 20-25, 2005), ed. S. Dimitrova. Heron Press, Sofia, p. 40 (2006) 